\title{
Weather-Based Optimal Power Flow with Wind Farms Integration
}

\author{
Jun Cao, Member, IEEE, W. Du, H. F. Wang, Senior Member, IEEE
}

\begin{abstract}
In conventional optimal power flow (OPF), the parameters of electrol components (e.g. resistance and thermal ratings of the overhead lines) are assumed to be constant despite the fact that they are strongly sensitive to the weather effect (e.g. temperature or wind speed) which influences the accuracy of optimal power flow results. This paper introduces a weatherbased optimal power flow (WB-OPF) algorithm with wind farm integration by considering the temperature related resistance and the dynamic line rating (DLR) of overhead transmission lines. A method of calculating the current-temperature relationship of bare overhead lines, given the weather conditions, is presented as a set of coupled temperature and power flow equations. A simplified general model is proposed to calculate the dynamic line rating (DLR) for maximizing the utilization of wind power. A Primal-dual Interior Point (PDIP) method is developed to solve the WB-OPF problem and the effectiveness of the proposed method is evaluated and demonstrated in the paper by two example power systems.
\end{abstract}

Index Terms - Electro-thermal Coupling, Dynamic Line Rating (DLR), Wind generation, weather effects, Weather-Based Optimal Power Flow (WB-OPF).

\section{NOMENCLATURE}

\section{Indices and Sets}

$i, j$

$k$

$k c$

$\mathbf{N}_{\mathbf{d c}}=\left\{1, \ldots, n_{d c}\right\}$

$\boldsymbol{C}=\{1, \ldots, c\}$

$U=\left\{P_{G j}^{\mathrm{MAX}}, P_{p c c i}^{\mathrm{MAX}}\right\}$

Indices.

State index ( $k=0$ denotes pre-contingency, $k>0$ denotes post-contingency).

Index of the most severe contingency.

The node set of the DC network.

Set of N-1 outage contingencies including both the traditional AC contingencies and outage of DC lines.

Set of control variables for Differential Evolution (DE) method.

Dr. Jun Cao, Dr. W. Du (corresponding author) and Prof. H. F. Wang are with the State Key Laboratory of Alternate Electrical Power System with Renewable Energy Sources, North China Electric Power University, Changping, Beijing, China.

This work was supported in part by the State Key Laboratory of Alternate Electrical Power System with Renewable Energy Sources, the National Basic Research Program of China (973Program) (2012CB215204), the National Natural Science Foundation of China (No. 51407070, 51311122), China, and in part by the Fundamental Research Funds for the Central Universities (No. 2014QN01), the project of State Grid Corporation of China (Grant: SGHB0000KXJS1400040)

$S_{C}^{P}$
$S_{C}^{C}$
$S_{U C}$
$S_{k c}$
$\mathrm{P}_{\mathrm{c}}^{*}$
Functions
$f, f_{0}$

$h_{0}, h_{k}^{0}, h_{k}$
$g_{0}, g_{k}^{0}, g_{k}$

Parameters

$W_{s}$

$d_{c}$

$U_{d c i}^{*}, P^{*}$

$P_{G j}^{\mathrm{MAX}}$

$P_{p c c i}^{\mathrm{MAX}}$

$h^{\mathrm{MAX}}$

$S_{\text {convi }}^{\mathrm{MAX}}$

$\Delta u_{s k}^{M A X}$

LineFlow $_{k}^{\mathrm{MAX}}$

$\alpha$

$r_{k c}$
Set of contingencies that are preventively controlled.

Set of contingencies that are correctively controlled.

Set of contingencies that cause infeasible solution.

Set of the most severe contingency.

Set of the SCOPF optimal solution.

Objective functions of the CSCOPF model with and without penalty term added.

Inequality constraints of the CSCOPF model for the base case and postcontingency short-term, long-term period. Equality constraints of the CSCOPF model for the base case and postcontingency short-term, long-term period.

Incident solar energy.

The conductor diameter.

The voltage and power references of the droop control strategy.

Upper limits of active power generation of the $j$ th unit (MW).

Upper limits of active power generation of the $i$ th converter (MW).

Vector of long-term flow limits.

Maximal apparent power of the $i$ th converter.

Maximal allowed adjustment variables of long-term control actions.

Maximal Line flow during the $k$ th contingency.

Parameter defining how much the shortterm post-contingency security constraints can be temporarily relaxed from the permanent limits.

Occurring probability of the $k c$ th contingency. 


$\begin{array}{ll}\lambda & \text { Penalty coefficient. } \\ Y_{d c i j} & \text { The element of bus admittance matrix } \mathbf{Y}_{\mathbf{d c}} \\ & \text { of the DC network. } \\ \text { NP } & \text { The number of the DE population number. } \\ \text { D } & \text { The dimension of the DE parameter } \\ & \text { vectors. }\end{array}$

Variables

$\begin{array}{cc}Q_{j} & \text { Heat gain due to resistive line losses }\left(P_{l o s s}[\mathrm{~W} / \mathrm{m}]\right) \\ Q_{s} & \text { Heat absorbed by solar radiation } \\ Q_{c} & \text { Forced convection heat loss } \\ Q_{r} & \text { Radiated heat loss } \\ & \text { period. }\end{array}$

\section{INTRODUCTION}

$\mathbf{I}_{\mathrm{p}}^{\mathrm{n}}$ ncread wind power generation has been connected to the power systems all over the world. These lead to significant challenges for the economical operation of power systems with large-scale wind power integration due to the stochastic characteristic of the wind speed. The variation nature of wind speed will introduce the changing of not only the wind power generation, but also the parameters of eletronic components (e.g. the resistance and thermal ratings of the overhead lines, as these parameters are strongly related to the weather effects, such as temperature, wind speed, et al. [1, 2]), which would affect allocating the system load power between conventional generators and wind-powered ones. Thus, there is a great need to incorporate these effects, bringing with the wind power integration, into the traditional OPF problem.

The variations of actual weather conditions will influence the resistances and thermal ratings of the system overhead lines and then affects the results of power flow and optimal power flow (OPF) [1, 2]. For example, the resistance of the power system equipment is a strong function of temperature and the line thermal rating varies with the weather conditions, such as the wind speed and direction, ambient temperature and solar radiation. However, traditional optimal power flow algorithms neglect the weather effects and take the resistance and thermal ratings of transmission lines as constant. These negligence will bring with two limitations: (1) some weatherrelated error is inherent in the OPF results by using the inaccurate resistance value [2]; (2) normally, the Dynamic (or real-time) thermal Line Rating (DLR) is higher than the static rating most of the time. Experience shows that an average of $50 \%$ extra capacity of overhear lines in favorable locations can be safely exploited by using the DLR technique [3]. Thus traditional OPF does not exploit the full capabilities of existing lines which results in higher cost of the total system. To accurately analysis the weather (mainly temperature, wind and solar radiation) related effect, this paper presents a weather-dependent optimal power flow technique to take account of the estimate of branch element weather factors in the wind power integrated OPF formulation.

In common practice, the steady state analysis such as power flow, optimal power flow and state estimation assume that the resistance of the overhead lines are constant and the calculation of system admittance was based on constant temperature (normally, the worst-case situation [1, 4]). However, the temperature, resistance and losses are interrelated and vary significantly in the real operation. Without considering the temperature-related variational part of resistance will lead to significant errors in loss estimations, especially under heavily loaded conditions [2]. To reduce the temperature-related error in the power flows analysis, Dr. Stephen Frank firstly introduces the temperature dependent power flow algorithm which integrates an estimate of branch temperatures and resistances with the conventional power flow equations [2, 5]. The work of [6] studies the influence of changes of the transmission lines resistance due to temperature on state estimation performance. The impact of transmission line temperature variations, resulting from loading and weather conditions changes, on system dynamic performance is analyzed in [7]. Although many research have focused on the environmental effect on system steady state and dynamic response, considering the weather related condition in the traditional optimal power flow algorithm with wind farms integration is still a blank field till now.

One of the key challenges faced during integration of wind powers with the grid is the spillage of wind energy due to the transmission constraints [8]. Many techniques have been introduced to minimize the spillage of wind power by using FACTs [9] and energy storage devices [10], which, however, is mostly cost expensive. The technology of Dynamic Line Rating (DLR) has attracted many attentions from the academic and industry, especially for maximizing the utilization of wind power [11]. Conventional static line rating (fixed summer/winter thermal rating) used in OPF is determined based on worst case weather assumption for operation. The analysis results are generally conservative and expensive. But in reality, the real capacity is not static and is a complex function of air temperature, solar radiation, local wind and actual current et al. [12]. One of the application example using the DLR technology is a leading UK distribution network which was concerned about network capacity problem when offshore and onshore wind farms were connected. Alstom Grid successfully alleviates the wind power integration problem by using the DLR technology. The trial showed that $50 \%$ or more wind generation could be connected to the grid compared to using the fixed summer/winter thermal ratings [11]. The work of [13] proposes a new general DLR calculation model and based on this, it develops an economic optimization simulation model regarding wind power integration by using a general DLR calculation model on overhead lines. The use of DLR technology will provide the true transfer capacity of the grid in real time accounting for actual weather conditions and improve the system reliability and utilization of the existing system [11].

This paper presents a novel treatment of the weatherdependent optimal power flow algorithm with wind farms integration which considers the temperature related resistance and the dynamic line rating (DLR) of overhead lines. A resistance-weather relationship and calculation modeling of 
DLR for overhead lines are present in Section II. Section III proposes the weather-based optimal power flow model with wind farms integration. A Primal-Dual Interior Point (PDIP) method is developed to solve the WB-OPF problem. Numerical solutions of two case studies using the MATLAB are discussed in Section IV. Finally, conclusions are drawn in Section V.

\section{WEATHER-DEPENDENT MODELING OF OVERHEAD LINES}

A. Resistance-weather relationship of bare overhead conductors

(1) Thermal balance of overhead lines

Fig. 1 illustrates the thermal balance model of overhead lines. The resistance of bare overhead conductors is a function of the ambient weather conditions according to the following steady state thermal balance equation [12]:

$$
Q_{j}+Q_{s}=Q_{c}+Q_{r}
$$

Fig. 1 Thermal balance model of overhead lines

The solar heat gain can be formulated as

$$
Q_{s}=W_{s} d_{c}
$$

The radiated heat loss $Q_{r}$ is nonlinear function of line temperature. However, it can be approximated as linear function of the conductor temperature rise over ambient [1]:

$$
\begin{aligned}
Q_{r} & =A_{r}\left[\left(T_{c}+273\right)^{4}-\left(T_{a}+273\right)^{4}\right] \\
& \approx K_{r} \cdot\left(T_{c}-T_{a}\right)
\end{aligned}
$$

Forced convection heat loss can be written as

$$
Q_{c}=K_{c} \cdot\left(T_{c}-\mathrm{T}_{a}\right)
$$

where $K_{r}$ and $K_{c}$ are the radiation and convective radiation heat transfer coefficients, respectively (equations are given in Appendix A).

Using (3) and (4), (1) can be rewritten as

$$
P_{\text {loss }}+W_{s} d_{c}=\left(K_{r}+K_{c}\right) \cdot\left(T_{c}-T_{a}\right)
$$

Thus, by rearranging (5)

$$
T_{c}=T_{a}+\left(P_{\text {loss }}+W_{s} d_{c}\right) \cdot \frac{1}{K_{r c}}
$$

Where $K_{r c}=K_{r}+K_{c}$ is the coefficient of heat loss.

(2) Resistance equation

The resistance of metallic conductors varies with the conductor temperature according to

$$
R_{c}=R_{a}\left[1+\alpha\left(T_{c}-T_{a}\right)\right]
$$

where

$R_{c}, R_{a} \quad$ conductor resistance at temperature $T_{c}$ and $T_{a}$

$T_{c}, T_{a} \quad$ conductor and ambient temperature $\left({ }^{\circ} \mathrm{C}\right)$

$\alpha \quad$ temperature coefficient

Fig. 2 represents the conductor temperature variation against the ambient temperature and wind speed. It shows that the wind speed, by changing the coefficient of $K_{c}$, is almost linear relationship with the conductor temperature.

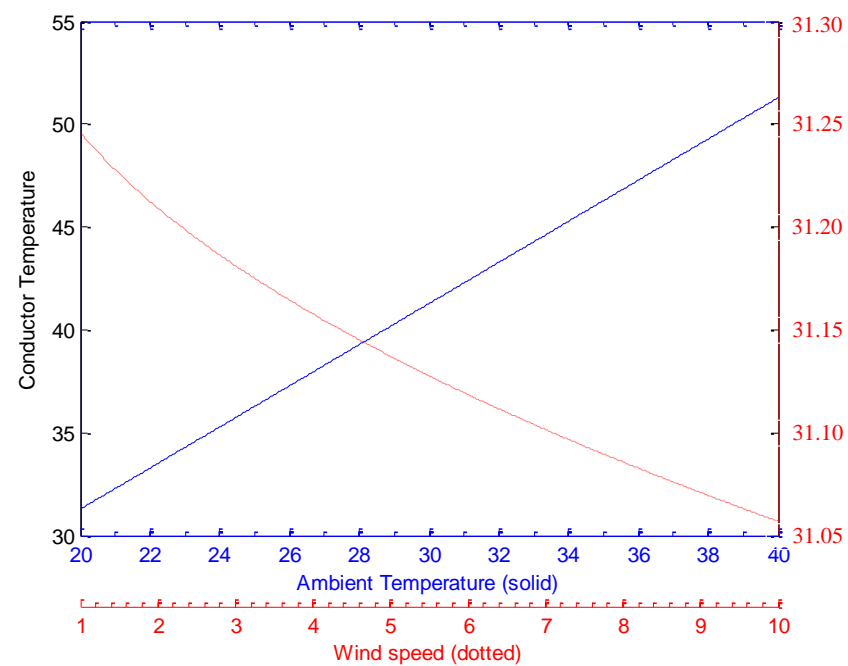

Fig. 2. Influence of the single parameter variation on the conductor temperature $T_{c}$

\section{B. Calculation modeling of Dynamic Line Rating (DLR)}

In reality, the transmission line rating are varied with: 1) current flowing in the conductor; 2) conductor size and resistance; 3) ambient weather conditions (temperature, wind speed and direction, solar radiation). A simple way to calculate the static line rating is based on the worst scenario. Engineering recommendation (ER) P27, which can be formulated as

$$
I_{\max }^{S L R}=\frac{\sqrt{\left[1.01+0.0371 \cdot\left(\frac{d_{c} \cdot \rho_{f} \cdot v^{S L R}}{\mu_{f}}\right)^{0.52}\right] \cdot\left[k_{f} \cdot K_{\text {angle }}^{S L R} \cdot\left(T_{c}-T_{a}\right)^{S L R}\right]}}{\sqrt{R^{S L R}}}
$$

where

$R^{S L R} \quad$ line resistance used in the scenario of SLR calculation

$v^{S L R} \quad$ wind speed in the SLR calculation

$\rho_{f}, \mu_{f}, k_{f}$ the density, dynamic viscosity and thermal conductivity of air

$K_{\text {angle }}^{\text {SLR }} \quad$ the angle between wind speed and the conductor axis in the scenario of SLR calculation

The Dynamic line rating (DLR) can be computed by using a variety of methods: conductor sag and tension monitoring, physical modeling and prediction techniques [13]. The simplified calculating model of DLR is based on the capacity ratio between DLR and SLR, which are given as below [13]:

$$
I_{\max }^{D L R}=\varepsilon_{v} \cdot \varepsilon_{T} \cdot I_{\max }^{S L R}
$$

where $\varepsilon_{v}$ and $\varepsilon_{T}$ are the ratios related to the wind speed and temperature, respectively.

Fig. 3 shows the dynamic line rating varies with the wind speed and ambient temperature. The current rating reaches maximum when wind speed is $10 \mathrm{~m} / \mathrm{s}$ and the temperature is $20^{\circ} \mathrm{C}$. Normally, when the wind speed is higher, the ambient temperature is lower, thus more wind powers can be transferred by using the modeling of dynamic line rating. 


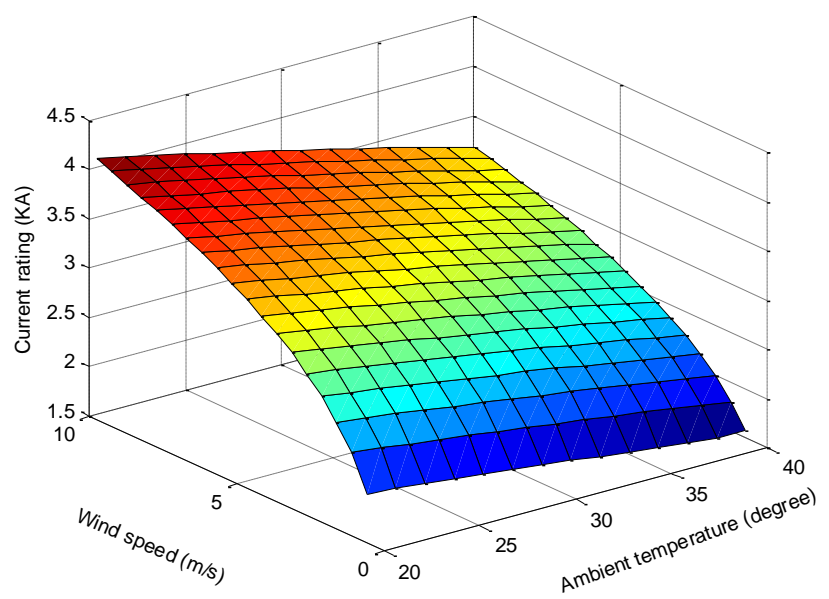

Fig. 3. Dynamic current rating versus wind speed and ambient temperature

\section{Formulation OF THE WEATHER-BASED OPF PROBLEM WITH WIND FARMS INTEGRATION}

\section{A. Weather-Based OPF Formulation}

To incorporate the weather condition into the traditional OPF problem with wind farm integration, the following modifications should be made:

1) the addition of branch temperatures to the vectors of system state variables;

2) the construction of a set of mismatch equations for thermal balance equations of overhead lines;

3) the incorporation of dynamic line rating model in the transmission line constraints;

4) the addition of wind generation cost to the objective functions;

5) the modeling of interdependence of temperature and the system via an augmented Jacobian and Hession matrix.

Fig. 4 shows a representation model of the weather-based OPF incorporating the wind farms which explains the state vectors and interactions of each module.

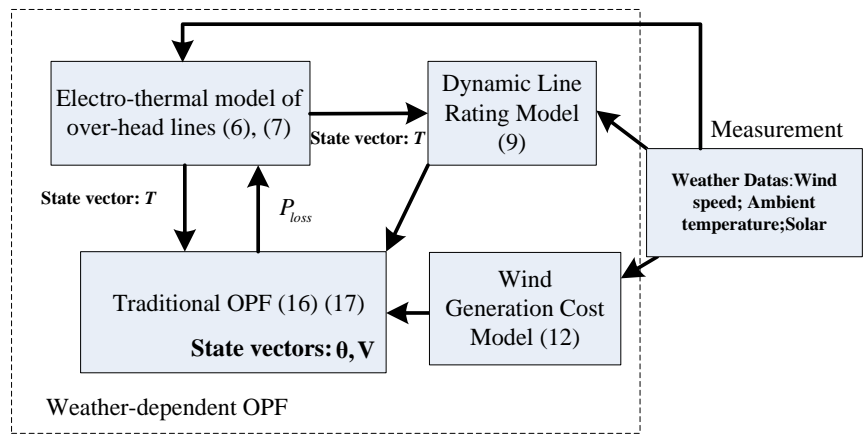

Fig. 4. Illustration of Weather-Based OPF model incorporation of Wind energy

Mathematically, the OPF can be stated as the following constrained nonlinear optimization problem [17]:

$$
\begin{array}{ll}
\text { Minimize } & \min f(\mathbf{y}) \\
\text { Subject to } & \boldsymbol{h}(\mathbf{y})=0 ; \boldsymbol{g}(\mathbf{y}) \leq 0 ; \underline{\mathbf{y}} \leq \mathbf{y} \leq \overline{\mathbf{y}}
\end{array}
$$

where $\min f(\cdot)$ is the objective function; the vector of $\mathbf{y}$ is the optimization variables which include the state variables $\mathbf{x}$ and control variables $\mathbf{u}$. The lower and upper limits of $\mathbf{y}$ are represented by $\underline{\mathbf{y}}$ and $\overline{\mathbf{y}}$, respectively. $\boldsymbol{h}(\mathbf{y})$ represents a set of equality constraints which includes both the nonlinear power flow mismatch equations and control equations. $g(\mathbf{y})$ is a set of inequality constraint functions to avoid the violation of the system limits.

\section{B. Wind Generation Cost Model}

The optimal schedule of wind farms output are highly dependent on the accuracy of wind power forecast technologies. However, the current forecast error from dayahead point prediction technique can be as high as $25 \% \sim 40 \%$ [14]. Therefore, it is necessary to include the forecast uncertainty into the objective cost function of OPF model.

The actual available wind power generation $\mathbf{P}_{\text {wind }}^{\text {Avai }}$ is a random variable which can hardly be predicted accurately. The probability density function (PDF) of $\mathbf{P}_{\text {Wind }}^{\text {Avai }}$ (or forecast error, forecast error $=\mathbf{P}_{\text {Wind }}^{\text {Ava }}$-forecast value) is a conditional probability function with respect to the forecast value [15]. Fig. 5 shows the distribution of wind power output $\mathbf{P}_{\text {Wind }}^{\text {Avai }}$ for a forecast value range $[0.20,0.24]$ by using the versatile probability distribution model in [15].

The integration of wind farms will introduce two types of generation cost: 1) wind spillage opportunity cost (green area in Fig. 5). Although there is no fuel cost for wind energy, the construction and operation of wind farms will introduce the initial investment and the maintenance costs, no matter whether the wind power is scheduled or not [16]. Thus wind power spillage, which refers to the amount of the unused wind power production ( $\left.\mathbf{P}_{\text {Wind }}^{\text {Avai }}-\mathbf{P}_{\text {Wind }}^{\text {Sche }}\right)$, will add extra opportunity cost to the objective function; 2) Reserve cost (red area in Fig. 5). When $\mathbf{P}_{\text {Wind }}^{\text {Avai }}$ is larger than $\mathbf{P}_{\text {Wind }}^{\text {Sche }}$, it will bring with extra reserve cost. Thus the expected wind generation cost can be defined as

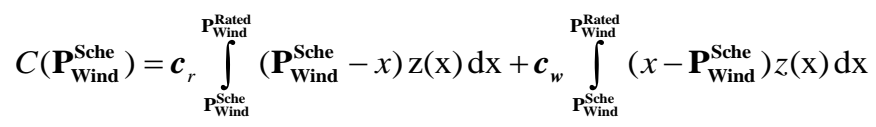

Where

$$
c_{w}=\frac{1}{\mathbf{C F}_{\text {Wind }}^{\text {design }}} \frac{C_{I}}{8760 n \mathbf{P}_{\text {wind }}^{\text {rated }}}\left\{1+m\left[\frac{(1+D)^{n}-1}{\mathrm{D}(1+D)^{n}}\right]\right\}
$$

$C_{I}$

CF $F_{\text {Wind }}^{\text {design }}$ $\mathbf{P}_{\text {wind }}^{\text {rated }}$

D

m

$n$

initial investment

capacity factor

rated wind power

discount rate

annual maintenance cost function

life time of plant 


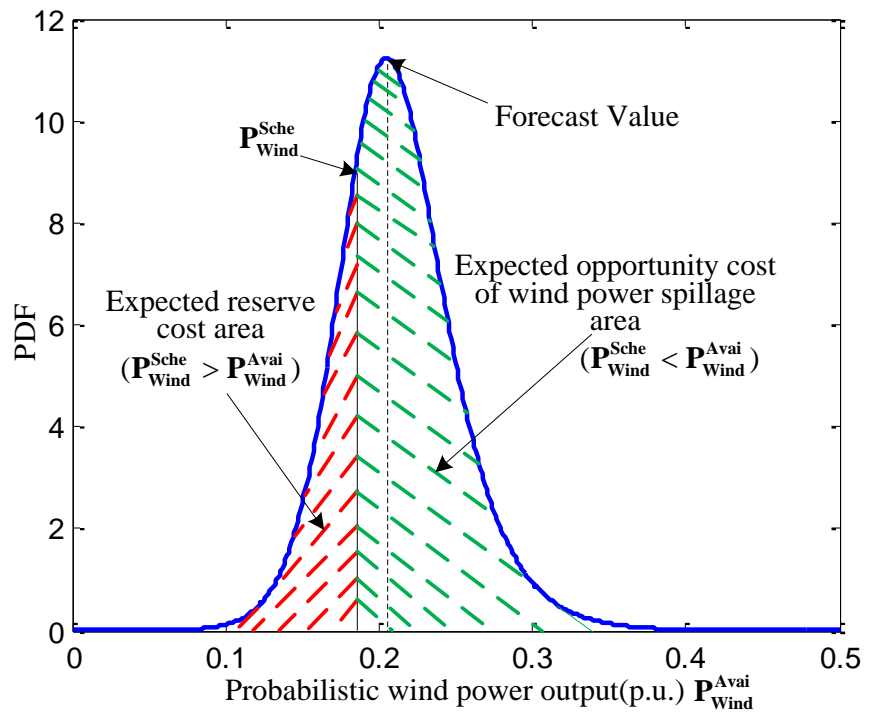

Fig. 5 Probabilistic distribution of wind power output for a given forecast value

$\mathbf{P}_{\text {Wind }}^{\text {Sche }} \quad$ the set of scheduled active power output of wind farms;

$\mathbf{P}_{\text {Wind }}^{\text {Rated }} \quad$ Rated active power output of wind farms;

$c_{w} \quad$ opportunity cost coefficient of wind power spillage, relating to under-forecasting of wind generated electricity [16];

$\boldsymbol{c}_{r} \quad$ reserve cost coefficient, relating to overforecasting of wind generated electricity;

$\mathrm{z}$ the probability distribution function (PDF) of wind power output $\mathbf{P}_{\text {Wind }}^{\text {Avai }}$;

\section{Objective Function with Wind Generation}

The main goal of objective function of the WB-OPF model with wind farms integration is to minimize the generation cost of the whole grid, meanwhile, reduce the opportunity cost and reserve cost of wind power. Hence the objective function can be written as

$$
\min f\left(\mathbf{P}_{\mathbf{G}}\right)=C_{g}\left(\mathbf{P}_{\mathbf{G}}\right)+C\left(\mathbf{P}_{\text {Wind }}^{\text {Sche }}\right)
$$

Where

$$
C_{g}\left(\mathbf{P}_{\mathbf{G}}\right)=c_{g 2} \mathbf{P}_{\mathbf{G}}^{2}+c_{g 1} \mathbf{P}_{\mathbf{G}}+c_{g 0}
$$

$C_{g} \quad$ cost function for the conventional generators

$C \quad$ penalty cost function for wind power forecasting error.

$\mathbf{P}_{\mathbf{G}} \quad$ the set of scheduled active power output of generators;

$c_{g 2}, c_{g 1}, c_{g 0}$ are the cost coefficients of generators.

It should be noted that the quantification of the wind generation cost is a complex process. Wind energy as a clean and environment-friendly technology will bring with social benefit, which are not include in the analysis.

\section{Equality and Inequality Constraints}

The equality constraints of WB-OPF include the conventional power flow mismatch equations and electrothermal coupling constrains which can be formulated as follows:

$$
\begin{aligned}
& P_{i}(\boldsymbol{\delta}, \mathbf{V}, \mathbf{T})=\sum_{i \in G N_{i}} P_{G i}+\sum_{i \in G W_{i}} P_{W i}-\sum_{i \in L N_{i}} P_{L i} \\
& =\sum_{j=1}^{N_{a}} V_{i} V_{j}\left[G_{i j}\left(T_{i j}\right) \cos \left(\delta_{i}-\delta_{j}\right)+B_{i j}\left(T_{i j}\right) \sin \left(\delta_{i}-\delta_{j}\right)\right] \forall i \in \mathbf{N}_{\mathbf{a c}} \\
& Q_{i}(\boldsymbol{\delta}, \mathbf{V}, \mathbf{T})=\sum_{j \in G N_{i}} Q_{G i}-\sum_{j \in C N_{i}} Q_{C i}-\sum_{j \in L N_{i}} Q_{L i} \\
& =\sum_{j=1}^{N_{a}} V_{i} V_{j}\left[G_{i j}\left(T_{i j}\right) \sin \left(\delta_{i}-\delta_{j}\right)-B_{i j}\left(T_{i j}\right) \cos \left(\delta_{i}-\delta_{j}\right)\right] \forall i \in \mathbf{N}_{\mathbf{a c}} \\
& H_{i j}(\boldsymbol{\delta}, \mathbf{V}, \mathbf{T})=T_{i j}- \\
& \left\{\begin{array}{l}
\left.T_{a}+\frac{1}{K_{r c, i j}}\left[G_{i j}\left(T_{i j}\right) \cdot\left(V_{i}^{2}+V_{j}^{2}\right)-2 G_{i j}\left(T_{i j}\right) \cdot V_{i} V_{j} \cos \left(\delta_{i}-\delta_{j}\right)\right]\right\} \\
\forall i, j \in \mathbf{N}_{\mathbf{a c}}
\end{array}\right.
\end{aligned}
$$

where $G_{i j}$ and $B_{i j}$ are the mutual (or self when $i=j$ ) conductance and mutual (or self when $i=j$ ) susceptance, respectively. $T_{\mathrm{ij}}$ and $T_{\mathrm{ij}, \max }$ are the conductor temperature and temperature limit of line $i-j$, respectively; $K_{r c, i j}$ is the heat loss coefficient of line $i-j . I_{W i \text { max }}$ is the branch thermal rating (static or dynamic).

Note that the optimization variables of the WB-OPF are $\mathbf{x}^{\mathbf{T}}=\left[\mathbf{U}, \boldsymbol{\theta}, \mathbf{T}, \mathbf{P}_{\mathbf{G}}, \mathbf{Q}_{\mathbf{G}}\right]$. Where, $\mathbf{U}$ is the bus voltage magnitude state variable vector, $\boldsymbol{\theta}$ bus voltage angle state variable vector, $\mathbf{T}$ conductor temperature state variable vector, $\mathbf{P}_{\mathbf{G}}$ active power generation control vector, $\mathbf{Q}_{\mathbf{G}}$ reactive power generation control vector, respectively.

To include the temperature related components in the WBOPF algorithm, the Jacobian and Hessian matrices need to be modified accordingly. Modification of the Jacobian and Hessian matrix is to add one state variable (temperature $\mathrm{T}$ ) per overhear line, one sets of equality constraints (Equation 18) and one sets of inequality constraints (Equation 22).

\section{Case Studies}

In this section, the WB-OPF algorithm is verified by use of a modified IEEE 9 node system and the New England transmission grid which are available in MATPOWER [17]. 
The two test systems have 9 and 46 branches respectively, however lossless branches (these with zero resistance) are excluded from the weather related analysis. All tests have been performed on a PC Intel Core i5-3470, 3.2-GHz, 4.00GB RAM.

To facilitate the algorithm stability and improve the converge process, the WB-OPF algorithm is initialized from a warm start. A temperature dependent power flow (TD-PF) algorithm proposed in [2] is adopted to calculate the WB-OPF initial value of voltage magnitude, angle and overhead line temperature.

A. Modified IEEE 9 node system integrated with a Wind Farm

\section{1) Weather based Optimal Power Flow Results}

The configuration of modified IEEE 9 bus system is shown in Fig. 6. All conductors are considered as hard-drawn aluminum with temperature coefficient $\alpha$ equals to 0.3951 and all conductors are initialized at a uniform ambient temperature. The maximum branch flow limit is set to be 100 MVA (the dynamic line rating is not considered in this test).

To allow comparison with traditional OPF problem, all the generators are assumed as thermal power generators and the generation cost coefficients $c_{g 2}, c_{g 1}, c_{g 0}$ are the same with the OPF in MATPOWER [17]. The primal-dual interior point (PDIP) method [17] is used to solve the WB-OPF problem and the algorithm converged successfully for all the test cases.

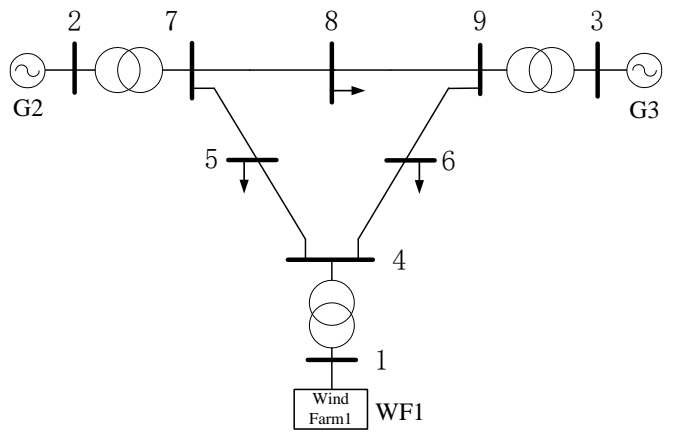

Fig. 6. Modified IEEE 9 node system

TABLE I

IMPACT OF TEMPERATURE ESTIMATION ON OPF RESULTS

\begin{tabular}{c|c|c}
\hline & OPF & WB-OPF \\
\hline Gen. Cost $(\$ / h r)$ & 5327.0 & 5290.7 \\
\hline$P_{W 1}(\mathrm{MW})$ & 97.28 & 89.85 \\
$P_{G 2}(\mathrm{MW})$ & 119.94 & 134.31 \\
$P_{G 3}(\mathrm{MW})$ & 100.90 & 94.17 \\
\hline Total losses & 3.1159 & 3.06518 \\
(MW) & & \\
\hline
\end{tabular}

Table II

DETAILED BRANCH RESUlTS FOR MODIFIED IEEE 9 NODE TEST SYSTEM

\begin{tabular}{|c|c|c|c|c|c|c|c|c|c|}
\hline \multirow[b]{2}{*}{ Branch } & \multirow[b]{2}{*}{$\begin{array}{l}\text { Power Flow } \\
\text { (MVA) }\end{array}$} & \multirow[b]{2}{*}{ Loading } & \multirow[b]{2}{*}{$\operatorname{Loss}(\mathrm{kW})$} & \multicolumn{3}{|c|}{ Resistance (p.u.) } & \multicolumn{3}{|c|}{ Temperature $\left({ }^{\circ} \mathrm{C}\right)$} \\
\hline & & & & OPF & $\begin{array}{l}\text { WB- } \\
\text { OPF }\end{array}$ & Change & $\begin{array}{c}\text { Ambient } \\
\left(T_{a}\right)\end{array}$ & $\begin{array}{l}\text { Conductor } \\
\left(T_{i j}\right)\end{array}$ & Change \\
\hline $1-4$ & 90.7887 & $36.32 \%$ & 0.00 & 0.0000 & 0.0000 & $0.00 \%$ & 5.00 & $-*$ & - \\
\hline $4-5$ & 37.9005 & $15.16 \%$ & 165.1 & 0.0170 & 0.0157 & $7.75 \%$ & 5.00 & 5.38 & $7.6 \%$ \\
\hline $5-6$ & 60.2024 & $40.13 \%$ & 940.9 & 0.0390 & 0.0363 & $6.87 \%$ & 5.00 & 7.60 & $52.0 \%$ \\
\hline $3-6$ & 98.0557 & $32.69 \%$ & 0.00 & 0.0000 & 0.0000 & $0.00 \%$ & 5.00 & - & - \\
\hline $6-7$ & 42.5446 & $28.36 \%$ & 137.9 & 0.0119 & 0.0110 & $7.40 \%$ & 5.00 & 6.26 & $25.2 \%$ \\
\hline $7-8$ & 64.3119 & $25.72 \%$ & 258.0 & 0.0085 & 0.0079 & $7.43 \%$ & 5.00 & 6.19 & $23.8 \%$ \\
\hline $8-2$ & 134.6352 & $53.85 \%$ & 0.00 & 0.0000 & 0.0000 & $0.00 \%$ & 5.00 & - & - \\
\hline $8-9$ & 74.5415 & $29.82 \%$ & 129.3 & 0.0320 & 0.0297 & $7.28 \%$ & 5.00 & 6.58 & $31.6 \%$ \\
\hline $9-4$ & 62.8247 & $25.13 \%$ & 270.2 & 0.0100 & 0.0093 & $7.48 \%$ & 5.00 & 6.06 & $21.2 \%$ \\
\hline
\end{tabular}

Table I shows the results of impact of temperature estimation on total system generation cost and branch losses (wind speed is set to constant $6 \mathrm{~m} / \mathrm{s}$ and ambient temperature equals $5^{\circ} \mathrm{C}$ ). It can be seen that inclusion of weather effect will influence the estimate of total system losses and decrease the generation cost by between one and two percent. The detailed branch flow, resistance and loss data for the modified IEEE 9 bus system are present in Table II. The differences in calculated conductor temperature are most pronounced for heavily loaded lines, for example, line 5-6 with $40.13 \%$ loading. The maximum increase in an individual branch resistance is $7.75 \%$ for line $4-5$.

Fig. 7 and Fig. 8 display the relationship of generation cost and total system losses with ambient temperature and wind speed, respectively. The system losses and generation cost will increase in proportion to the ambient temperature, and decrease with more wind power injected into grid. As higher ambient temperature and lower wind speed will lead to increasing of branch resistance according to (1) and (7) which promoting the system losses and generation cost. 


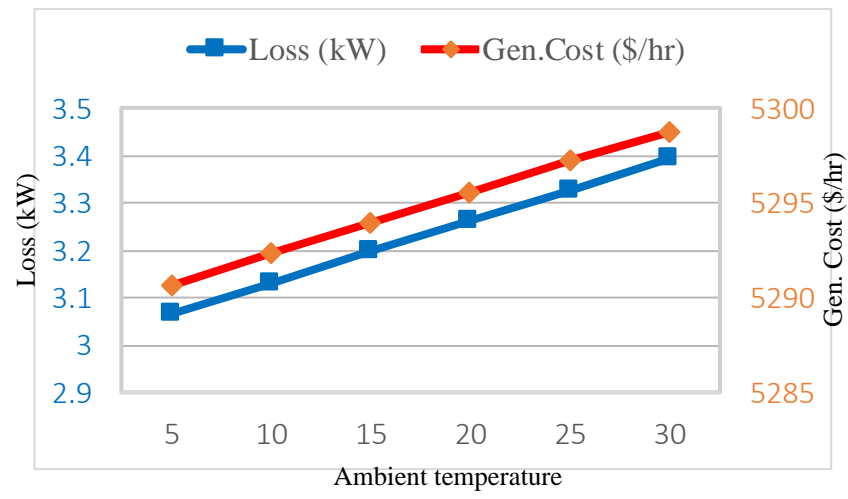

Fig. 7 Generation cost and system losses versus ambient temperature (wind speed $=6 \mathrm{~m} / \mathrm{s}$ )

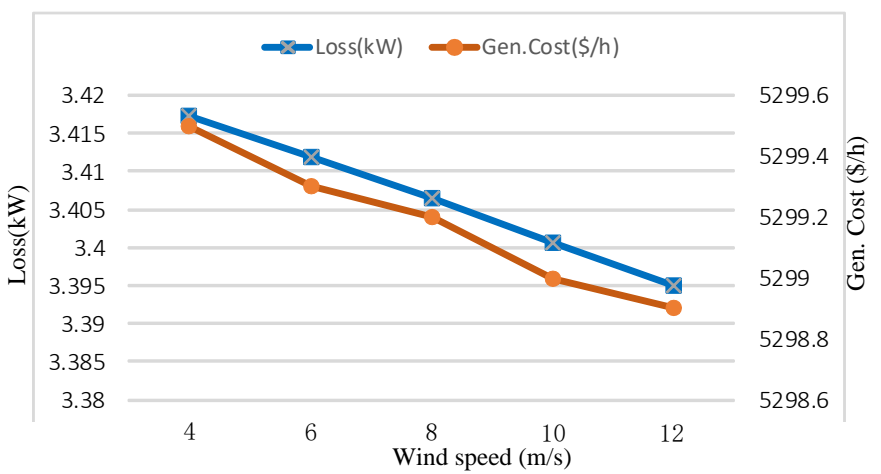

Fig. 8 Generation cost and system losses versus wind speed (ambient temperature $=25{ }^{\circ} \mathrm{C}$ )

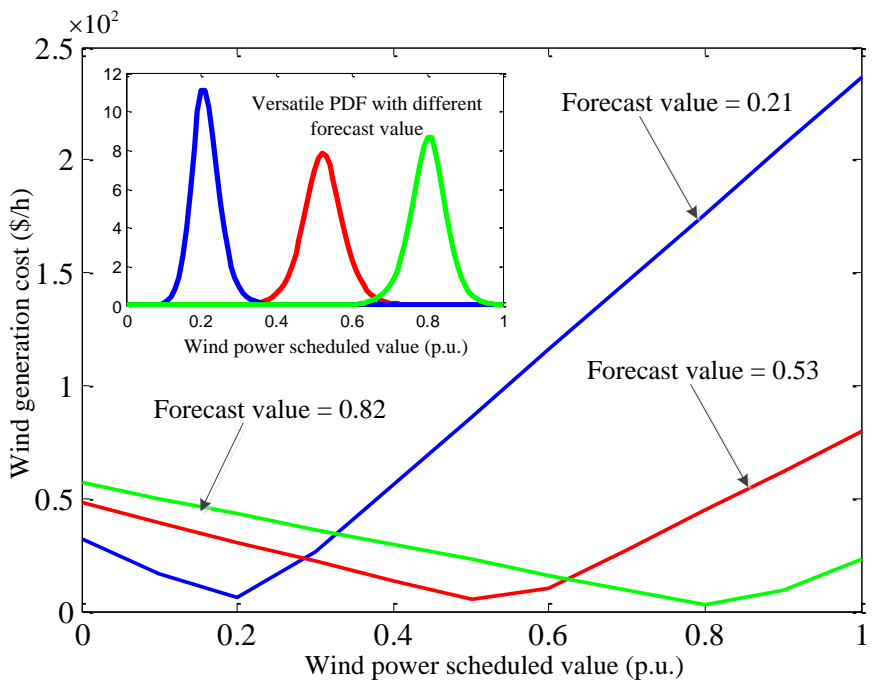

Fig. 9 Wind generation cost $C\left(\mathbf{P}_{\text {Wind }}^{\text {Sche }}\right)$ versus scheduled wind power for different wind forecast value

\section{2) WB-OPF with wind farm integration}

To analysis the impact of wind farm integration, the traditional generator G1 is replaced by one wind farm (WF1). The parameters of wind farm are shown in Table V, Appendix B. The cost coefficient of wind generated electricity $\boldsymbol{c}_{w}$ is obtained from (13) as $40 \$ / \mathrm{MWh}$ and the reserve cost coefficient $\boldsymbol{c}_{r}$ is set to $3 \$ / \mathrm{MWh}$. The proposed WB-OPF algorithm converged successfully for all test cases.

Fig. 9 shows the versatile PDF of wind power output and the variations of wind generation cost calculated by (12) with different scheduled wind power $\mathbf{P}_{\text {Wind }}^{\text {Sche }}$ for forecast value equal $0.21,0.53$ and 0.82 . It is found that the point of minimum wind generation cost almost near the forecast value.

Table III shows the WB-OPF results with different wind power forecast value. With the increasing of wind forecast value, the WB-OPF solution is scheduling more powers for wind plant at WF 1 . As the generation cost of wind power are comparatively lower compared with thermal generators. Thus, the total generation cost are substantial decreasing by $73.3 \%$ with the increased scheduled wind powers.

Comparison results with and without dynamic line rating are illustrated in Fig. 10. The dynamic line rating of overhead lines 4-5 in IEEE 9 bus system (black solid line) are increasing with the wind speed. Simulation results show that the DLR technique can help increase the transfer limits of overhead lines by approximately $50 \%$. It can decrease the generation cost (black line shadow saving area) by $69.8 \%$ and reduce the wind power spillage (red line shadow area) by $35 \%$ which can maximize the utilization of green energy.

TABLE III

WB-OPF RESULTS WITH DIFFERENT FORECAST VALUE

\begin{tabular}{c|ccc|cc}
\hline $\begin{array}{c}\text { Wind } \\
\text { forecast } \\
\text { value (p.u.) }\end{array}$ & $\begin{array}{c}\mathrm{P}_{\mathrm{G} 2} \\
(M W)\end{array}$ & $\begin{array}{c}\mathrm{P}_{\mathrm{G} 3} \\
(M W)\end{array}$ & $\begin{array}{c}\mathrm{P}_{\mathrm{W} 1} \\
(M W)\end{array}$ & $\begin{array}{c}\text { Wind } \\
\text { gen. cost } \\
(\$ / h r)\end{array}$ & $\begin{array}{c}\text { Total Gen. } \\
\text { cost } \\
(\$ / h r)\end{array}$ \\
\hline 0.04 & 183.6 & 128.5 & 10 & 10.7 & 6182.6 \\
0.3 & 141.8 & 99.39 & 77.5 & 48.6 & 4172.6 \\
0.6 & 95.28 & 67.05 & 155.0 & 26.8 & 2465.5 \\
0.9 & 63.39 & 44.87 & 227.5 & 5.3 & 1649.4 \\
\hline
\end{tabular}

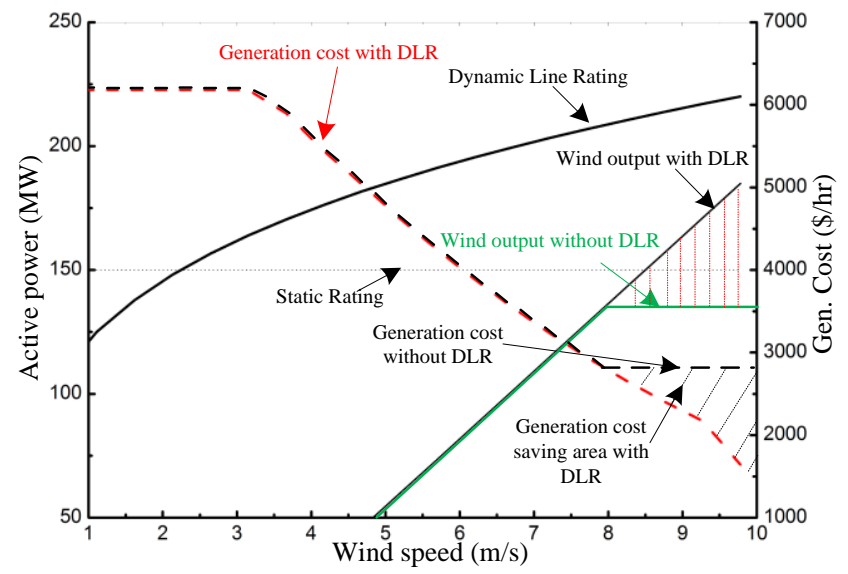

Fig. 10 Comparison results with and without dynamic line rating

\section{B. New England 39 bus test system}

The modified New England 39 bus system is used to test the proposed algorithm. This system has 10 units, 46 branches, and 39 buses. The traditional generator G1, G2, G3 are replaced by three wind farms (WF1, WF2, and WF3).

The primal-dual interior point (PDIP) method [17] is used to solve the WB-OPF problem and the algorithm converged successfully. The computation results of the OPF, WB-OPF and TD-PF [2] are compared in Table IV. It can be seen that inclusion of weather effect will change the estimate of total 
system losses, generation cost and improve the estimation accuracy of branch resistances and losses.

TABLE IV RESULTS OF THE WB-OPF ALGORITHM

\begin{tabular}{c|ccc}
\hline & $O P F$ & $W B-O P F$ & $T D-P F^{*}$ \\
\hline Gen. Cost & 41864.18 & 41884.47 & - \\
$(\$ / \mathrm{hr})$ & & & \\
$P_{W 1}(\mathrm{MW})$ & 671.59 & 675.62 & 250.00 \\
$P_{W 2}(\mathrm{MW})$ & 646.00 & 626.00 & 679.18 \\
$P_{W 3}(\mathrm{MW})$ & 671.16 & 673.66 & 650.00 \\
Total losses & 43.60 & 45.04 & 44.95 \\
$(\mathrm{MW})$ & & & \\
\hline
\end{tabular}

*Temperature dependent power flow in [2]

\section{PRACTICAL IMPLEMENT CONSIDERATIONS}

The implementation of WB-OPF algorithm in practice needs that the weather condition (e.g. wind speed, ambient temperature and solar radiation) throughout the power grid can be obtained through sensors in real time operation or be predicted by weather forecast model in day ahead implementation which are shown in Fig. 11.

The real-time implementation of WB-OPF requires high resolutions and wide-area measurement of the ambient weather condition across the system. However, in the lack of detailed wide-are sensor data, several weather zones around the available sensor points can be created to approximate the ambient weather [2].

The proposed method is a centralized WB-OPF algorithm. So the high-speed communication technology is a big issue that counteracts its real-time application. However, communication technology is developed at a very fast rate and a wide-area communication system is possible in the future.

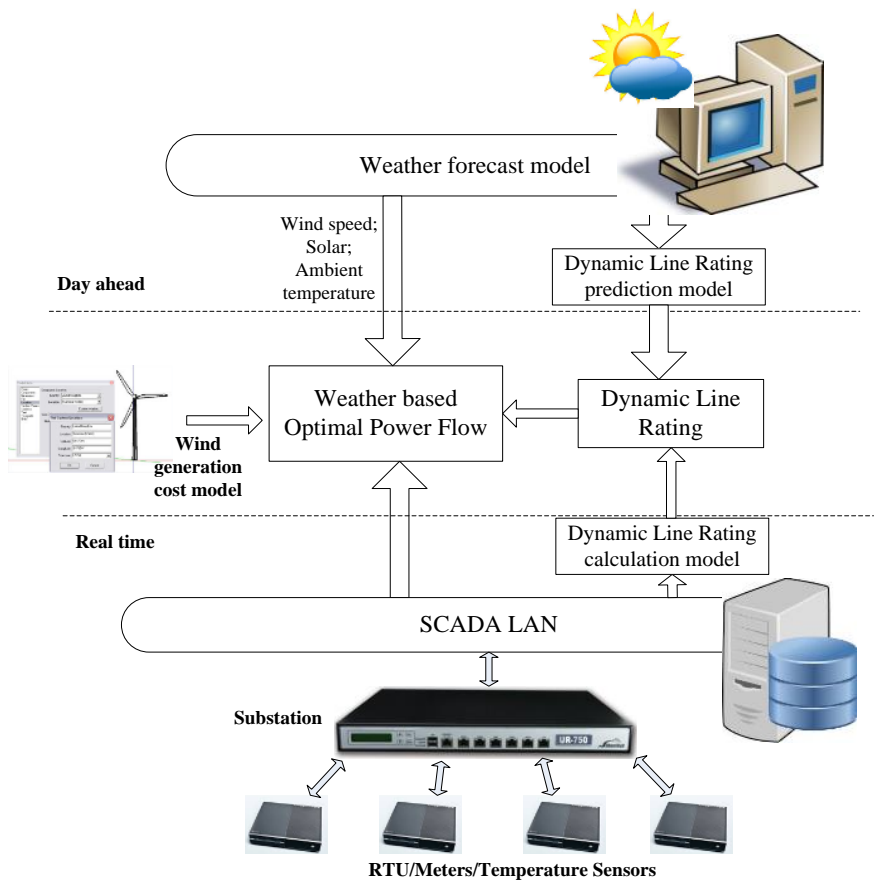

Fig. 11 Illustration of WB-OPF practical implement

\section{CONCLUSION}

Traditional computation of optimal power flow neglects the electro-thermal coupling of overhead lines. The paper proposes a weather-based OPF model with wind farm integration by considering the temperature related resistance and the dynamic line rating (DLR) of overhead transmission lines. This WB-OPF formulation was successfully applied to two examples. Simulation results of implementing the WBOPF demonstrated that

(1) The resulting of WB-OPF algorithm can improve estimation accuracy of branch resistances and losses compared with traditional OPF. Furthermore, it can potentially reduce the total generation costs.

(2)Weather-based OPF by using Dynamic Line Rating (DLR) helps to manage and optimize power generation according to the actual dynamic or real time thermal rating of the overhead lines by taking account the weather conditions.

(3)The DLR technique can maximize the usable capacity of overhead line assets dramatically, avoid upgrading or replacing existing transmission lines (saving significant capital investment) and can potentially reduce the wind power spillage.

The proposed method could also be used to analysis weather dependent distributed generation such as wind power and PV, together with more advanced integrated techniques in power systems motivates models that consider weather.

\section{REFERENCES}

[1] J.R. Santos, A. Gómez Expósito and F. Parreño Sánchez, "Assessment of conductor thermal models for grid studies," IET Gen. Transm. Distrib., vol. 1, no. 1, pp. 155-161, Jan. 2007.

[2] Stephen Frank, Jason Sexauer and Salman Mohagheghi, "TemperatureDependent Power Flow," IEEE Tans. Power Syst., vol. 28, no. 4, pp. 4007-4018, Nov., 2013.

[3] Ampacimon. Innovative solution for dynamic line rating. [Online]. Available: https://www.ampacimon.com/?dl_id=34

[4] IEEE Recommended Practice for Industrial and Commercial Power Systems Analysis, IEEE Std. 399, 1997.

[5] S. Frank, J. Sexauer, and S. Mohagheghi, Temperature Dependent Power Flow Code, 2013. [Online]. Available: https://github.com/TDPF/TDPF.

[6] M. Bočkarjova and G. Andersson, "Transmission line conductor temperature impact on state estimation accuracy," in Proc. Powertech 2007 IEEE Lausanne, Jul. 2007, pp. 701-70.

[7] Aseem Rambani, Paul Lerley and Larry Fish, "Impact of weather condition on steady state and dynamic response of power system," in North American Power Symposium (NAPS), Sep. 2012.

[8] S.J. Plathottam, P. Ranganathan and H. Salehfar, "Unbiased Optimal Power Flow for Power Systems with Wind Power Generaion," IEEE Electronics Letters, vol. 50, no. 18, pp. 1312-1314, Aug., 2014.

[9] Amin Nasri, Antonio J. Conejo, S. Jalal Kazempour, and Mehrdad Ghandhari, "Minimizing Wind Power Spillage Using an OPF With FACTS Devices," IEEE Trans. on Power Syst., vol. 29, no. 5, pp. 21502159, Sep., 2014.

[10] M. Ghofrani, A. Arabali, M. Etezadi-Amoli, and M. S. Fadali, "A framework for optimal placement of energy storage units within a power system with high wind penetration," IEEE Trans. Sustain. Energy, vol. 4, no. 2, pp. 434-442, Apr., 2013.

[11] MiCOM ALSTOM P341, Dynamic line rating Brochure GB. [Online]. Available: http://www.alstom.com/Global/Grid/Resources/Documents/A utomation/SAS/MiCOM\%20ALSTOM\%20P341\%20DLR.pdf. 
[12] D. R. Swtek, "An expeted per-unit rating for overhead transmission lines," Int. J. Elect. Power Energy Syst., vol. 26, no. 4, pp. 241-247, May, 2004.

[13] Carl Johan Wallnerström, Yalin Huang and Lennart Sder, "Impact From Dynamic Line Rating on Wind Power Integration," IEEE Trans. On Smart Grid, Early review, 2014.

[14] T. Ackermann, Wind Power in Power System. London, U.K.: Wiley, 2005.

[15] Zhao-Sui Zhang, Yuan-Zhang Sun, David Wenzhong Gao, Jin Lin and Lin Cheng, "A Versatile Probability Distribution Model for Wind Power Forecast Errors and Its Application in Economic Dispatch," IEEE Tans. Power Syst., vol. 28, no. 3, pp. 3114-3125, Aug., 2013.

[16] Sathyajith, M. "Wind energy: fundamentals, resource analysis and economics,"Springer, Vol. 28, No. 3, pp. 3401-3409, Aug. 2006.

[17] R. D. Zimmerman, C. E. Murillo-Sánchez, and R. J. Thomas, "MATPOWER: Steady-State Operations, Planning and Analysis Tools for Power Systems Research and Education," IEEE Tans. Power Syst., vol. 26, no. 1, pp. 12-19, Feb. 2011.

\section{APPENDIX A}

The equations of radiation and convective radiation heat transfer coefficients are:

$$
\begin{gathered}
K_{c}=K_{\phi} k_{f}\left(T_{c}-\mathrm{T}_{a}\right)\left[1.01+0.371\left(\frac{D \rho_{f} V_{w}}{\mu_{f}}\right)^{0.52}\right] \\
K_{r}=0.318 D \varepsilon \frac{\left(T_{c}+273\right)^{4}-\left(T_{a}+273\right)^{4}}{100^{4}}
\end{gathered}
$$

where $K_{\phi}=1.194-\cos (\phi)+0.194 \cos (2 \phi)+0.368 \sin (2 \phi)$ is a term that accounts for the angle $\phi$ between the wind direction and conductor axis,

The equations of $\varepsilon_{v}$ and $\varepsilon_{T}$ are expressed as:

$$
\begin{aligned}
& \varepsilon_{v}=\max \\
& \left\{\begin{array}{c}
1 \\
v_{w}^{0.26} / v_{S L R}^{0.26} \\
\frac{0.566}{v_{S L R}^{0.26}}\left(\frac{\rho_{f}}{\mu_{f}}\right)^{0.04} \cdot D^{0.04} \cdot v^{0.3} \\
\varepsilon_{T}=\sqrt{\frac{T_{c}-T_{a}}{T_{c}-T_{A}^{S L R}}}
\end{array}\right.
\end{aligned}
$$

where $D=0.028 v_{S L R}=0.5 T_{A}^{S L R}=35$

\section{APPENDIX B}

TABLE V THE PARAMETERS OF WIND TURBINE

\begin{tabular}{|c|c|c|c|c|c|}
\hline$C_{I}$ & $\mathbf{C F}_{\text {Wind }}^{\text {design }}$ & $\mathbf{P}_{\text {wind }}^{\text {rated }}$ & $D$ & $m$ & $n$ \\
\hline$\$ 550000$ & 0.25 & $600 \mathrm{KW}$ & 0.05 & 0.035 & $\begin{array}{c}20 \\
\text { years }\end{array}$ \\
\hline
\end{tabular}

